Submitted to the Annals of Statistics

\title{
SUPPLEMENTARY PROOFS: ASYMPTOTIC THEORY FOR DENSITY RIDGES
}

\author{
By Yen-Chi Chen, Christopher R. Genovese and Larry \\ WASSERMAN \\ Carnegie Mellon University
}

This paper contains the proofs to the lemmas in the paper 'Asymptotic Theory for Density Ridges'. To be consistent, we keep the numbering for each lemma and theorem the same as the original paper.

\section{Proofs.}

Lemma 1. Assume the matrix $M(x)$ has rank $d-1$. Then $e(x)$, the first eigenvector of

$$
\mathbf{I}_{d}-M(x)\left(M(x)^{T} M(x)\right)^{-1} M(x)^{T},
$$

is tangent to $R_{h}$ at $x \in R_{h}$. The column space of $M(x)$ is normal to $R_{h}$ at each $x \in R_{h}$.

Proof For Lemma 1. Let $M(x)=\left(m_{2}(x), \cdots, m_{d}(x)\right)$ with each $m_{k}(x)$ being the column vector for $M(x)$ and $V_{h}(x)=\left[v_{2}(x), \cdots, v_{d}(x)\right]$. Note that we begin with $m_{2}(x)$ in the matrix $M(x)$. Then

$$
\begin{aligned}
R_{h} & =\left\{x: V_{h}(x)^{T} g_{h}(x)=0, \lambda_{2}(x)<0\right\} \\
& =\bigcap_{k=2}^{d}\left\{x: v_{k}(x)^{T} g_{h}(x)=0, \lambda_{2}(x)<0\right\} .
\end{aligned}
$$

By definition,

$$
\begin{aligned}
M(x)=\nabla\left(V_{h}(x)^{T} g_{h}(x)\right) & =\left[\nabla\left(v_{2}(x)^{T} g_{h}(x)\right), \cdots, \nabla\left(v_{d}(x)^{T} g_{h}(x)\right)\right] \\
& =\left(m_{2}(x), \cdots, m_{d}(x)\right) .
\end{aligned}
$$

We conclude that $m_{k}(x)=\nabla\left(v_{2}(x)^{T} g_{h}(x)\right)$.

The gradient $\nabla f(x)$ is normal to the set of a contour surface $\{x: f(x)=$ $0\}$ for each $x \in\{x: f(x)=0\}$. As a result, $m_{k}(x)$ is normal to $\{x$ : $\left.v_{k}(x)^{T} g_{h}(x)=0, \lambda_{2}(x)<0\right\}$ whenever $x \in\left\{x: v_{k}(x)^{T} g_{h}(x)=0, \lambda_{2}(x)<\right.$ $0\}$. Since the ridge set is intersection of all $\left\{x: v_{k}(x)^{T} g_{h}(x)=0, \lambda_{2}(x)<0\right\}$, for any $x \in R_{h}$, the tangent vector of $R_{h}$ at $x$ must be normal to each 
$m_{k}(x), k=2, \cdots, d$. This proves that the tangent direction of $R_{h}$ at $x \in R_{h}$ is normal to column space of $M(x)$.

As $M(x)$ has rank $d-1, M(x)^{T} M(x)$ is invertible so $e(x)$ exists. Note $M(x)\left(M(x)^{T} M(x)\right)^{-1} M(x)^{T}$ is the projection matrix onto the column space of $M(x)$. The matrix

$$
\mathbf{I}_{d}-M(x)\left(M(x)^{T} M(x)\right)^{-1} M(x)^{T}
$$

is the projection onto the subspace normal to the column space of $M(x)$. Hence, the first eigenvector, $e(x)$, must be normal to the column space of $M(x)$. This completes the proof.

Lemma 2 (Properties of the normal space). Let $q$ be a density that satisfies (A1) and (P1) and denote $R_{q}=\operatorname{Ridge}(q)$. Let $R_{q}^{\delta}=R_{q} \oplus \delta_{0}$ where $\delta_{0}$ is the gap defined in (P1). Let $M_{q}(x), N_{q}(x)$ be constructed from equation (34) in section 3.2. Then

1. $N_{q}$ and $M_{q}$ have the same column space. Also,

$$
N_{q}(x) N_{q}(x)^{T}=M_{q}(x)\left[M_{q}(x)^{T} M_{q}(x)\right]^{-1} M_{q}(x)^{T} .
$$

That is, $N_{q}(x) N_{q}(x)^{T}$ is the projection matrix onto columns of $M_{q}(x)$.

2. The columns of $N_{q}(x)$ are orthonormal to each other.

3. For $x \in R_{q}$, the column space of $N_{q}(x)$ is normal to the direction of $R_{q}$ at $x$.

4. For all $x \in R_{q}, \operatorname{rank}\left(N_{q}(x)\right)=\operatorname{rank}\left(M_{q}(x)\right)=d-1$. Moreover, $R_{q}$ is a 1-dimensional manifold that contains no intersection and no endpoints. Namely, $R_{q}$ is a finite union of connected, closed curves.

5. When $\|x-y\|$ is sufficiently small and $x, y \in R_{q}^{\delta}$,

$\left\|N_{q}(x) N_{q}(x)^{T}-N_{q}(y) N_{q}(y)^{T}\right\|_{\max } \leq A_{0}\left(\left\|q^{(3)}\right\|_{\infty}+\left\|q^{(4)}\right\|_{\infty}\right)^{2}\|x-y\|$,

for some constant $A_{0}$.

6. Assume $q^{\prime}$ also satisfies (A1), (P1) and $\left\|q-q^{\prime}\right\|_{\infty, 3}^{*}$ is sufficiently small. Then

$$
\left\|N_{q}(x) N_{q}(x)^{T}-N_{q^{\prime}}(x) N_{q^{\prime}}(x)^{T}\right\|_{\max } \leq A_{1}\left(\left\|q-q^{\prime}\right\|_{\infty, 3}^{*}\right)
$$

for some constant $A_{1}$.

7. The reach of $R_{q}$ satisfies

$$
\operatorname{reach}\left(R_{q}\right) \geq \min \left\{\frac{\delta_{0}}{2}, \frac{\beta_{2}^{2}}{A_{2}\left(\left\|q^{(3)}\right\|_{\infty}+\left\|q^{(4)}\right\|_{\infty}\right)}\right\}
$$

for some constant $A_{2}$.

imsart-aos ver. 2013/03/06 file: ridgeAOSsupp.tex date: March 10, 2015 
Proof For Lemma 2. Because $N_{q}(x)$ is the QR-factorization of $M_{q}(x)$, claim 1-2 are trivially true since (P1) ensures that $M_{q}(x)$ exists (but may not be unique) for $R_{q}^{\delta}$. To prove claim 3 , note that by claim 1 the column space of $N_{q}(x)$ is the same as $M_{q}(x)$. By Lemma 1, column space of $M_{q}(x)$ is normal to the direction of $R_{q}$ at $x \in R_{q}$ which proves the assertion.

The proofs for Claims 4 to 6 are more involved. We first derive the form of $M_{q}(x) M_{q}(x)$ and show that it depends on $\lambda_{1}(x)$ and the projection matrix $V(x) V(x)^{T}$ and $H_{0}(x)=H(x)-\lambda_{1}(x) v_{1}(x) v_{1}(x)^{T}$.

Recall that $M_{q}(x)=\left[m_{2}(x), \cdots, m_{d}(x)\right]$ where

$$
\begin{aligned}
m_{k}(x) & =\left(\lambda_{k}(x) \mathbf{I}_{d}+\frac{v_{1}(x)^{T} g_{q}(x)}{\lambda_{k}(x)-\lambda_{1}(x)} \nabla_{v_{1}(x)} H_{q}(x)\right) v_{k}(x) \\
& =\left(\lambda_{k}(x) \mathbf{I}_{d}+\frac{1}{\lambda_{k}(x)-\lambda_{1}(x)} T(x)\right) v_{k}(x)
\end{aligned}
$$

and $T(x)=v_{1}(x)^{T} g_{q}(x) \nabla_{v_{1}(x)} H_{q}(x)$. Let

$$
\begin{aligned}
& \Lambda_{0}(x)=\operatorname{Diag}\left(\lambda_{2}(x), \cdots, \lambda_{d}(x)\right) \\
& \Lambda_{1}(x)=\operatorname{Diag}\left(\frac{1}{\lambda_{2}(x)-\lambda_{1}(x)}, \cdots, \frac{1}{\lambda_{d}(x)-\lambda_{1}(x)}\right)
\end{aligned}
$$

where $\operatorname{Diag}\left[a_{1}, \cdots, a_{k}\right]$ is a diagonal matrix with element $a_{1}, \cdots, a_{k}$. Note that $\Lambda_{0}(x), \Lambda_{1}(x)$ are $(d-1) \times(d-1)$. Recall that $V(x)=\left[v_{2}(x), \cdots, v_{d}(x)\right]$. Then

$$
M_{q}(x)=\left[m_{2}(x), \cdots, m_{d}(x)\right]=V(x) \Lambda_{0}(x)+T(x) V(x) \Lambda_{1}(x) .
$$

Now

$$
\begin{aligned}
M_{q}(x) M_{q}(x)^{T}= & V(x) \Lambda_{0}(x)^{2} V(x)^{T}+T(x) V(x) \Lambda_{1}(x) \Lambda_{0}(x) V(x)^{T} \\
& +V(x) \Lambda_{0}(x) \Lambda_{1}(x) V(x)^{T} T(x)+T(x) V(x) \Lambda_{1}(x)^{2} V(x)^{T} T(x) .
\end{aligned}
$$

We used the fact that $T(x), \Lambda_{1}(x)$ are symmetric. Since $V(x) \Lambda_{0}(x) V(x)^{T}$ and $V(x) \Lambda_{0}(x) \lambda_{1}(x) V(x)^{T}$ are symmetric and $V(x)$ has orthonormal columns, we can rewrite the above as

$$
\begin{aligned}
M_{q}(x) M_{q}(x)^{T} & =\left(V(x) \Lambda_{0}(x) V(x)^{T}+V(x) \Lambda_{1}(x) V(x)^{T} T(x)\right)^{2} \\
& =\left(H_{0}(x)+H_{1}(x) T(x)\right)^{2}
\end{aligned}
$$

where

$$
\begin{aligned}
& H_{0}(x)=V(x) \Lambda_{0}(x) V(x)^{T} \\
& H_{1}(x)=V(x) \Lambda_{1}(x) V(x)^{T} .
\end{aligned}
$$


Note that $H_{0}(x)+H_{0}(x) H_{1}(x) T(x)$ is a symmetric matrix.

Proof for claim 4. Because we use condition (P1) frequently in this proof, we restate it here:

(P1) There exists constants $\beta_{0}, \beta_{1}, \beta_{2}, \delta_{0}>0$ such that

$$
\begin{aligned}
\lambda_{2}(x) & \leq-\beta_{1} \\
\lambda_{1}(x) & \geq \beta_{0}-\beta_{1} \\
\left\|g_{h}(x)\right\| \max _{|\alpha|=3}\left|p_{h}^{(\alpha)}(x)\right| & \leq \beta_{0}\left(\beta_{1}-\beta_{2}\right)
\end{aligned}
$$

for all $x \in R_{h} \oplus \delta_{0}$.

Note that $\beta_{1}-\beta_{2}>0$.

We first prove that $M_{q}(x) M_{q}(x)^{T}$ has constant rank $d-1$ for all $x \in R_{q}^{\delta}$. By (5),

$$
M_{q}(x) M_{q}(x)^{T}=\left(H_{0}(x)+H_{1}(x) T(x)\right)^{2} .
$$

We need to show that $H_{0}(x)+H_{1}(x) T(x)$ always has rank $d-1$. Recall $T(x)=v_{1}(x)^{T} g_{q}(x) \nabla_{v_{1}(x)} H_{q}(x)$ so that by $(\mathrm{P} 1)$,

$$
\|T(x)\|_{\max } \leq \beta_{0}\left(\beta_{1}-\beta_{2}\right) .
$$

Note that each eigenvalue in $H_{1}(x)$ has absolute values $\leq \frac{1}{\beta_{0}}$ by $(\mathrm{P} 1)$. This implies that all eigenvalues for $H_{1}(x) T(x)$ are in $\left[-\beta_{1}+\beta_{2}, \beta_{1}-\beta_{2}\right]$. (P1) also require all eigenvalues for $H_{0}(x)$ be less than $-\beta_{1}<0$. Thus, the $d-1$ smallest eigenvalues value of $H_{0}(x)+H_{1}(x) T(x)$ must be $\leq-\beta_{2}<0$ which shows the rank of $M_{q}(x) M_{q}(x)^{T}$ is $d-1$.

Thus, by the implicit function theorem, $R_{q}$ is a 1-dimensional manifold. That is, $R_{q}$ is a union of curves. This directly implies that $R_{q}$ cannot have intersections (two or more curves intersecting each others) otherwise the intersected point will violate the rank condition.

The last step is to prove that $R_{q}$ cannot have endpoints. We prove this by contradiction. We assume that $R_{q}$ has an end point $x_{0}$. We have shown that $M_{q}(x)$, the derivative over $V_{q}(x)^{T} g_{q}(x)$, is bounded. Thus, $x_{0}$ must be in $R_{q}$. To see this, we consider $V_{q}\left(x_{0}\right)^{T} g_{q}\left(x_{0}\right)$ and $\lambda_{2}\left(x_{0}\right)$. We must have $V_{q}\left(x_{0}\right)^{T} g_{q}\left(x_{0}\right)=0$ since $x_{0}$ is the end point of $R_{q}$ and $V_{q}(x)^{T} g_{q}(x)$ has derivative $M_{q}(x)$ which is bounded near $R_{q} . \lambda_{2}\left(x_{0}\right)$ must be negative since $x_{0} \in R_{q}^{\delta}$. Thus, $x_{0} \in R_{q}$. However, this contradicts to the implicit function theorem since at the end point $x_{0} \in R_{q}$, we cannot find a smooth local 
parameterization for $R_{q}$ defined on an open set. Accordingly, we conclude that $R_{q}$ have no end point.

Proof for claim 5. We prove that $H_{0}(x), H_{1}(x)$ are smooth functions of the Hessian. Let

$$
H_{1}^{*}(x)=H_{0}(x)-\lambda_{1}(x) V(x) V(x)^{T} .
$$

Then

$$
\begin{aligned}
& H_{1}(x) H_{1}^{*}(x) H_{1}(x) \\
& \quad=H_{1}(x) V(x)\left(\Lambda_{0}(x)-\lambda_{1}(x) \mathbf{I}_{d-1}\right) V(x)^{T} V(x) \Lambda_{1}(x) V(x)^{T} \\
& =H_{1}(x) V(x) V(x)^{T}=H_{1}(x)
\end{aligned}
$$

and similarly $H_{1}^{*}(x) H_{1}(x) H_{1}^{*}(x)=H_{1}(x)$. Thus, $H_{1}^{*}(x)$ is the pseudo inverse of $H_{1}(x)$. Note that $H_{1}^{*}(x)$ always has rank $d-1$ for $x \in R_{q}^{\delta}$ by the eigengap $\beta_{0}$ from (P1). By definition, $H_{1}(x)$ has constant rank $d-1$ for $x \in R_{q}^{\delta}$, hence, $H_{1}^{*}(x)$ uniquely determine $H_{1}(x)$ and depends only on $H_{0}(x), \lambda_{1}(x)$ and $V(x) V(x)^{T}$.

It is well-known that $\lambda_{1}(x)$ is differentiable and has bounded derivative because of the eigengap. Hence, $\nabla \lambda_{1}(x)$ exists and is uniformly bounded for all $x \in R_{q}^{\delta}$.

To bound $V(x) V(x)^{T}$ and $H_{0}(x)$, we use the matrix perturbation theory in Bhatia (1997); Chen et al. (2003). Let $\mathcal{H}$ be the collection of all $d \times d$ symmetric matrices and $f: \mathbb{R} \mapsto \mathbb{R}$. For a matrix $H \in \mathcal{H}$ with spectral decomposition $H=U \Lambda U^{T}$ where $\Lambda=\operatorname{Diag}\left(\mu_{1}, \cdots, \mu_{d}\right)$. We define a matrixvalued function $f_{\square}: \mathcal{H} \mapsto \mathcal{H}$ generated by $f$ as

$$
f_{\square}(H)=U \operatorname{Diag}\left(f\left(\mu_{1}\right), \cdots, f\left(\mu_{d}\right)\right) U^{T} .
$$

The function $f_{\square}$ is well-defined and inherits all the smoothness conditions that $f$ has. In this case, from Bhatia (1997), we have that, for a small symmetric perturbation $\Delta_{\epsilon}$,

$$
\left\|f_{\square}(H)-f_{\square}\left(H+\Delta_{\epsilon}\right)\right\|_{\max }=\|f\|_{\infty}^{(1)}\left\|\Delta_{\epsilon}\right\|_{\max }+o\left(\left\|\Delta_{\epsilon}\right\|_{\max }\right)
$$

where $\|f\|_{\infty}^{(1)}$ is the uniform bound for derivative of $f$.

Observe that $H_{0}(x)=f_{\square, 0}\left(H_{q}(x)\right), V(x) V(x)^{T}=f_{\square, 1}\left(H_{q}(x)\right)$ where $f_{\square, l}$ 
is generated by $f_{l}$ with

$$
\begin{aligned}
& f_{0}(x)= \begin{cases}0 & \text { if } x \geq-\beta_{1}+\beta_{0} \\
x & \text { if } x \leq-\beta_{1}\end{cases} \\
& f_{1}(x)= \begin{cases}0 & \text { if } x \geq-\beta_{1}+\beta_{0} \\
1 & \text { if } x \leq-\beta_{1}\end{cases}
\end{aligned}
$$

and for $x \in\left[-\beta_{1},-\beta_{1}+\beta_{0}\right]$, we can always choose a smooth function to link them.

By (9),(10), there exists constants $\gamma_{0}, \gamma_{1}$ depending only on $\beta_{0}, \beta_{1}$ such that, if $\left|H_{q}(x)-H_{q}(y)\right|$ is small,

$$
\begin{aligned}
\left\|H_{0}(x)-H_{0}(y)\right\|_{\max } & \leq \gamma_{0}\left\|H_{q}(x)-H_{q}(y)\right\|_{\max } \\
\left\|V(x) V(x)^{T}-V(y) V(y)^{T}\right\|_{\max } & \leq \gamma_{1}\left\|H_{q}(x)-H_{q}(y)\right\|_{\max } .
\end{aligned}
$$

Since $\lambda_{1}(x)$ has bounded derivative and $H_{1}(x), H_{1}^{*}(x)$ have constant rank $d-1$, and the pseudo-inverse $H_{1}^{*}(x)$ is Lipschitz, we have that $H_{1}(x)$ is Lipschitz by Theorem 3.3 in page 12 in (Stewart, 1977) that is,

$$
\left\|H_{1}(x)-H_{1}(y)\right\|_{\max } \leq \gamma_{2}\left\|q^{(3)}\right\|_{\infty}\left\|H_{q}(x)-H_{q}(y)\right\|_{\max }
$$

for some constant $\gamma_{2}$ depending on $\beta_{0}, \beta_{1}$. The term $\left\|q^{(3)}\right\|_{\infty}$ comes from the first eigenvalue. Thus, we have shown that both $H_{0}, H_{1}$ are Lipschitz.

Recall from $(5), M_{q}(x) M_{q}(x)^{T}=\left(H_{0}(x)+H_{1}(x) T(x)\right)^{2}$. Thus, we need to show $T(x)$ is also Lipschitz continuous. $T(x)$ is the derivatives for $H_{q}(x)$ along $v_{1}(x) ; v_{1}(x)$ is a uniquely defined and differentiable vector. By Taylor's theorem, $T(x)$ is Lipschitz with constant proportional to $\|q\|_{\infty}^{(4)}$. Putting this all together, we conclude that for any $x, y \in R_{q}^{\delta}$ that are sufficiently close,

$$
\left\|M_{q}(x) M_{q}(x)^{T}-M_{q}(y) M_{q}(y)^{T}\right\|_{\max } \leq \gamma_{4}\left(\left\|q^{(3)}\right\|_{\infty}+\left\|q^{(4)}\right\|_{\infty}\right)\|x-y\|
$$

for some constant $\gamma_{4}$ depending on $\beta_{0}, \beta_{1}$.

The last step is to bound $N_{q}(x) N_{q}(x)^{T}$. Note that $N_{q}(x) N_{q}(x)^{T}=U_{q}(x) U_{q}(x)^{T}$ where $U_{q}(x)=\left[u_{2}(x), \cdots, u_{d}(x)\right]$ where each $u_{k}(x)$ is an eigenvector of $M_{q}(x) M_{q}(x)^{T}$ corresponding to the ordered eigenvalues. We apply the DavisKahan theorem so that

$$
\begin{aligned}
&\left\|N_{q}(x) N_{q}(x)^{T}-N_{q}(y) N_{q}(y)^{T}\right\|_{\max } \leq \frac{d^{2}\left\|M_{q}(x) M_{q}(x)^{T}-M_{q}(y) M_{q}(y)^{T}\right\|_{\max }}{\beta_{2}} \\
& \leq \gamma_{5}\left(\left\|q^{(3)}\right\|_{\infty}+\left\|q^{(4)}\right\|_{\infty}\right)\left\|H_{q}(x)-H_{q}(y)\right\|_{\max } \\
& \leq \gamma_{5}\left(\left\|q^{(3)}\right\|_{\infty}+\left\|q^{(4)}\right\|_{\infty}\right)^{2}\|x-y\|, \\
& \text { imsart-aos ver. 2013/03/06 file: ridgeAOSsupp.tex date: March 10, } 2015
\end{aligned}
$$


for some constant $\gamma_{5}$ depending only on $\beta_{0}, \beta_{1}, \beta_{2}$. Note that we use Taylor's theorem to conclude that $\left\|H_{q}(x)-H_{q}(y)\right\|_{\max } \leq\left\|q^{(3)}\right\|_{\infty}\|x-y\|$. This completes the proof of Claim 4.

Proof for claim 6. The proof for Claim 6 is essentially the same as the proof for Claim 5. We replace $M_{q}(y)$ by $M_{q^{\prime}}(x)$ and repeat the argument above. Let $H_{0}^{\prime}(x), H_{1}^{\prime}(x), T^{\prime}(x)$ denote the counterparts of $H_{0}(x), H_{1}(x), T(x)$ for $M_{q^{\prime}}(x)$. Namely,

$$
M_{q^{\prime}}(x) M_{q^{\prime}}(x)^{T}=\left[H_{0}^{\prime}(x)+H_{1}^{\prime}(x) T^{\prime}(x)\right]^{2}
$$

The difference $\left\|H_{0}^{\prime}(x)-H_{0}(x)\right\|_{\max }$ or $\left\|H_{1}^{\prime}(x)-H_{1}(x)\right\|_{\max }$ are less than or equal to $O\left(\left\|q-q^{\prime}\right\|_{\infty, 2}^{*}\right)$ by the matrix perturbation theory. $\left\|T(x)-T^{\prime}(x)\right\|_{\max }$ is bounded by $O\left(\left\|q-q^{\prime}\right\|_{\infty, 3}^{*}\right)$. Hence, the total difference can be bounded by $\gamma_{6}\left\|q-q^{\prime}\right\|_{\infty, 3}^{*}$ for some constant $\gamma_{6}$.

Proof for claim 7. The following proof is a modification of the proof of Lemma 4.11 in Federer (1959). Let $r_{0}=\min \left\{\frac{\delta_{0}}{2}, \frac{\beta_{2}^{2}}{K}\right\}$, where $K=\gamma_{6}\left(\left\|q^{(3)}\right\|_{\infty}+\right.$ $\left.\left\|q^{(4)}\right\|_{\infty}\right)$ for some constant $\gamma_{6}$. We show that $r_{0}$ is a lower bound for $\operatorname{reach}\left(R_{q}\right)$ by considering $x$ such that $d\left(x, R_{q}\right)<r_{0}$ and we prove $x$ has unique projection onto $R$.

We recall the following two facts:

(F1) $M_{q}(x) M_{q}(x)^{T}$ is Lipschitz in $x \in R_{q}^{\delta}$ in the following sense

$$
\left\|M_{q}(x) M_{q}(x)^{T}-M_{q}(y) M_{q}(y)^{T}\right\|_{2} \leq K\|x-y\|
$$

for sufficiently small $\|x-y\|$. This follows from (13). Note that in (13), we use the max norm but here we apply $L_{2}$ norm so that the constant $\gamma_{4}$ is replaced by $\gamma_{6}$.

(F2) For any vector $v \in \mathbb{R}^{d}$ in the column space of $M_{q}(x)$,

$$
\frac{\left\|M_{q}(x) M_{q}(x)^{T} v\right\|}{\|v\|} \geq \beta_{2}^{2} .
$$

This follows from the fact that in proving claim 4, we have shown that the first $d-1$ eigenvalues of $M_{q}(x) M_{q}(x)^{T}$ are greater or equal to $\beta_{2}^{2}$.

We prove this claim by contradiction. Assume $x$ has multiple projection onto $R_{q}$ and $d\left(x, R_{q}\right)<r_{0}$.

imsart-aos ver. 2013/03/06 file: ridgeAOSsupp.tex date: March 10, 2015 
Without loss of generality, let $b, c \in R_{q}$ be two points that are projections of $x$. Since $b, c \in R_{q}, f(b)=f(c)=0$ and by Taylor's theorem with (F1),

$$
\begin{aligned}
\left\|(b-c)^{T} M_{q}(b) M_{q}(b)^{T}\right\| & =\left\|f(b)-f(c)-(b-c)^{T} M_{q}(b) M_{q}(b)^{T}\right\| \\
& \leq \frac{1}{2}\|b-c\|^{2} K .
\end{aligned}
$$

We can find a vector $t_{b} \in \mathbb{R}^{d}$ and $t_{b}$ is in column space of $M_{q}(x)$ such that $x-b=M_{q}(b) M_{q}(b)^{T} t_{b}$. Together with (14),

$$
\begin{aligned}
2\left|(b-c)^{T}(x-b)\right| & =2\left|(b-c)^{T} M_{q}(b) M_{q}(b)^{T} t_{b}\right| \\
& \leq\left\|(b-c)^{T} M_{q}(b) M_{q}(b)^{T}\right\|\left\|t_{b}\right\| \\
& \leq K\|b-c\|^{2}\left\|t_{b}\right\| .
\end{aligned}
$$

Since $b, c$ are projection of $x$ onto $M,\|x-b\|=\|x-c\|$. As a result,

$$
\begin{aligned}
0 & =\|x-c\|^{2}-\|x-b\|^{2} \\
& =\|b-c\|^{2}+2(b-c)^{T}(x-b) \\
& \geq\|b-c\|^{2}-K\|b-c\|^{2}\left\|t_{b}\right\| \quad \text { by }(15) \\
& =\|b-c\|^{2}\left(1-K\left\|t_{b}\right\|\right) .
\end{aligned}
$$

Now since $t_{b}$ is in column space of $M_{q}(b)$, by (F2)

$$
\frac{\beta_{2}^{2}}{K}>r_{0} \geq\|x-b\| \geq\left\|M_{q}(b) M_{q}(b)^{T} t_{b}\right\| \geq \beta_{2}^{2}\left\|t_{b}\right\|
$$

This shows that $K\left\|t_{b}\right\|<1$ and hence $\|b-c\|^{2} \leq 0$ by (16) so that $b=c$. Thus, $r_{0}$ is a lower bound for the reach.

Lemma 4. Assume (P1-2). Let

$$
W(x)=N(x)^{T} H_{N}^{-1}(x) N(x)^{T}=N(x)^{T}\left(N(x)^{T} H(x) N(x)\right)^{-1} N(x)^{T} .
$$

\section{Then:}

1. For any other $d \times(d-1)$ matrix $N^{\prime}(x)$ such that $N^{\prime}(x)^{T} N^{\prime}(x)=\mathbf{I}_{d-1}$ and $N^{\prime}(x) N^{\prime}(x)^{T}=N(x) N(x)^{T}$, $N(x)^{T}\left(N(x)^{T} H(x) N(x)\right)^{-1} N(x)^{T}=N^{\prime}(x)^{T}\left(N^{\prime}(x)^{T} H(x) N^{\prime}(x)\right)^{-1} N^{\prime}(x)^{T}$ when $x \in R_{h} \oplus \delta_{0}$. 
2. When $\|x-y\|$ is sufficiently small,

$$
\|W(x)-W(y)\|_{\max } \leq A_{3}\left(\left\|q^{(3)}\right\|_{\infty}+\left\|q^{(4)}\right\|_{\infty}\right)^{2}\|x-y\|
$$

for some constant $A_{3}$.

3. Assume another density $q$ satisfies (A1) and (P1) and let $W_{q}(x)$ be the counterpart of $W(x)$ for density $q$. When $\left\|p_{h}-q\right\|_{\infty, 3}^{*}$ is sufficiently small,

$$
\left\|W(x)-W_{q}(x)\right\|_{\max } \leq A_{4}\left\|p_{h}-q\right\|_{\infty, 3}^{*}
$$

for some constant $A_{4}$.

Proof For Lemma 4. Observe that the matrix $W(x)$ has pseudo-inverse

$$
W^{*}(x)=N(x) N(x)^{T} H(x) N(x) N(x)^{T} .
$$

Note that both $W(x), W^{*}(x)$ have rank $d-1$ for all $x \in R_{h} \oplus \delta_{0}$. Hence, $W(x)$ is uniquely determined by its pseudo-inverse $W^{*}(x)$.

Claim 1 follows from the fact that the pseudo inverse depends only on $N(x) N(x)^{T}$ and $H(x)$.

To prove claim 2 , by claim 5 of Lemma 2 ,

$$
\begin{gathered}
\|H(x)-H(y)\|_{\max } \leq \gamma_{7}\left\|p_{h}^{(3)}\right\|_{\infty}\|x-y\| \\
\left\|N(x) N(x)^{T}-N(y) N(y)^{T}\right\|_{\max } \leq A_{0}\left(\left\|p_{h}^{(3)}\right\|_{\infty}+\left\|p_{h}^{(4)}\right\|_{\infty}\right)^{2}\|x-y\|,
\end{gathered}
$$

for some constants $\gamma_{7}, A_{0}$. Thus, by Theorem 3.3, page 12 in Stewart (1977) the pseudo-inverse is Lipschitz in the following sense:

$$
\left\|W^{*}(x)-W^{*}(y)\right\|_{\max } \leq A_{3}\left(\left\|p_{h}^{(3)}\right\|_{\infty}+\left\|p_{h}^{(4)}\right\|_{\infty}\right)^{2}\|x-y\|
$$

for some constant $A_{3}$. This proves claim 2 .

Claim 3 follows from the similar way as claim 2; note that we apply claim 6 in Lemma 2 for different densities.

Lemma 9. Let $R$ be the ridge of a density $p$. For $x \in R$, let the Hessian at $x$ be $H(x)$ with eigenvectors $\left[v_{1}, \cdots, v_{d}\right]$ and eigenvalues $0>\lambda_{2} \geq \cdots \lambda_{d}$. Consider any subspace $\mathbb{L}$ spanned by a basis $\left[e_{2}, \cdots e_{d}\right]$ with $e_{1}$ in the normal direction of that subspace. Then a sufficient condition for $x$ being a local mode of $p$ constrained to $\mathbb{L}$ is

$$
\left(v_{1}^{T} e_{1}\right)^{2}>\frac{\lambda_{1}}{\lambda_{1}-\lambda_{2}}
$$

imsart-aos ver. 2013/03/06 file: ridgeAOSsupp.tex date: March 10, 2015 
Proof For Lemma 9. We assume $\lambda_{1} \geq 0$ otherwise this is trivially true.

Now we consider $x \in R$ and the Hessian matrix $H(x)=U(x) \Omega(x) U^{T}(x)$, where $U(x)=\left(v_{1}(x), \cdots, v_{d}(x)\right), \Omega=\operatorname{diag}\left(\lambda_{1}(x), \cdots, \lambda_{d}(x)\right)$ is the eigenvector/eigenvalue matrix from spectral decomposition. For abbreviation, we write $U \equiv U(x), \Omega \equiv \Omega(x)$ and $v_{i} \equiv v_{i}(x), \lambda_{i} \equiv \lambda_{i}(x), i=1, \cdots, d$.

Let $L=\left(e_{2}, \cdots, e_{d}\right)$ be the matrix whose column space is $\mathbb{L}$. All we need to show is that $\nabla_{L} \nabla_{L} p(x)=L^{T} H(x) L$ is negative definite. This is equivalent to prove that for any $\alpha=\left(\alpha_{2}, \cdots, \alpha_{d}\right) \in \mathbb{R}^{d-1}$,

$$
\alpha^{T} L^{T} U \Omega U^{T} L \alpha<0
$$

when $\left(v_{1}^{T} e_{1}\right)^{2}>\frac{\lambda_{1}}{\lambda_{1}-\lambda_{2}}$.

Let $\beta_{j i}=e_{j}^{T} v_{i}$ for $i, j=1, \cdots, d$.

$$
\begin{aligned}
\alpha^{T} L^{T} U \Omega U^{T} L \alpha & =\sum_{i=1}^{d} \sum_{j=2}^{d} \alpha_{j}^{2} \beta_{j i}^{2} \lambda_{i} \\
& =\sum_{i=2}^{d} \sum_{j=2}^{d} \alpha_{j}^{2} \beta_{j i}^{2} \lambda_{i}+\sum_{j=2}^{d} \alpha_{j}^{2} \beta_{j 1}^{2} \lambda_{1} \\
& \leq \sum_{j=2}^{d} \alpha_{j}^{2} \lambda_{2} \sum_{i=2}^{d} \beta_{j i}^{2}+\sum_{j=2}^{d} \alpha_{j}^{2} \beta_{j 1}^{2} \lambda_{1} .
\end{aligned}
$$

Note that we use the fact $\lambda_{i} \leq \lambda_{2}, \forall i=3, \cdots, d$ in the last inequality.

Since $\beta_{j i}=e_{j}^{T} v_{i}$ and $v_{1}, \cdots, v_{d}$ spanned the whole $\mathbb{R}^{d}$,

$$
\sum_{i=1}^{d} \beta_{j i}^{2}=\left\|e_{j}\right\|^{2}=1 .
$$

Thus, $\sum_{i=2}^{d} \beta_{j i}^{2}=1-\beta_{j 1}^{2}$. We can rewrite (22) as

$$
\begin{aligned}
\alpha^{T} L^{T} U \Omega U^{T} L \alpha & \leq \sum_{j=2}^{d} \alpha_{j}^{2} \lambda_{2}\left(1-\beta_{j 1}^{2}\right)+\sum_{j=2}^{d} \alpha_{j}^{2} \beta_{j 1}^{2} \lambda_{1} \\
& =\sum_{j=2}^{d} \alpha_{j}^{2}\left(\lambda_{2}\left(1-\beta_{j 1}^{2}\right)+\lambda_{1} \beta_{j 1}^{2}\right) .
\end{aligned}
$$

Since $\beta_{j 1}^{2}=e_{j}^{T} v_{1}$ and $e_{1}, \cdots, e_{d}$ spanned $\mathbb{R}^{d}$, we conclude $1=\sum_{j=1}^{d} \beta_{j 1}^{2}$ so that $\beta_{j 1}^{2} \leq 1-\beta_{11}=1-e_{1}^{T} v_{1}$. Now by definition, $\lambda_{2}<0$ and $\lambda_{1} \geq 0$, the 
equation (24) can be upper-bounded by

$$
\begin{aligned}
\alpha^{T} L^{T} U \Omega U^{T} L \alpha & \leq \sum_{j=2}^{d} \alpha_{j}^{2}\left(\lambda_{2}\left(1-\beta_{j 1}^{2}\right)+\lambda_{1} \beta_{j 1}^{2}\right) \\
& \leq \sum_{j=2}^{d} \alpha_{j}^{2}\left(\lambda_{2}\left(e_{1}^{T} v_{1}\right)^{2}+\lambda_{1}\left(1-e_{1}^{T} v_{1}\right)^{2}\right) \\
& =\left(\lambda_{2}\left(e_{1}^{T} v_{1}\right)^{2}+\lambda_{1}\left(1-e_{1}^{T} v_{1}\right)^{2}\right) \times \sum_{j=2}^{d} \alpha_{j}^{2} .
\end{aligned}
$$

Therefore, as long as

$$
\lambda_{2}\left(e_{1}^{T} v_{1}\right)^{2}+\lambda_{1}\left(1-e_{1}^{T} v_{1}\right)^{2}<0,
$$

we conclude that $p$ is the local mode in the subspace of $\mathbb{L}$. After rearrangement, equation (26) is equivalent to

$$
\left(v_{1}^{T} e_{1}\right)^{2}>\frac{\lambda_{1}}{\lambda_{1}-\lambda_{2}}
$$

so that we have completed the proof.

Lemma 10. (Gine and Guillou (2002); version of Genovese et al. (2012)) Assume (K1-K2) and that $\log n / n \leq h^{d} \leq b$ for some $0<b<1$. Then we have

$$
\left\|\widehat{p}_{n}-p\right\|_{\infty, k}=O\left(h^{2}\right)+O_{P}\left(\sqrt{\frac{\log n}{n h^{d+2 k}}}\right)
$$

for $k=0, \cdots, 4$. In particular, if we consider the smoothed version of density, $p_{h}$, for the same kernel function, then we have

$$
\left\|\widehat{p}_{n}-p_{h}\right\|_{\infty, k}=O_{P}\left(\sqrt{\frac{\log n}{n h^{d+2 k}}}\right)
$$

for $k=0, \cdots, 4$.

Theorem 12 (Theorem 6 in Genovese et al. (2012)). Assume condition (A1),(P1) for two densities $p_{1}, p_{2}$. When $\left\|p_{1}-p_{2}\right\|_{\infty, 3}^{*}$ is sufficiently small, we have $\operatorname{Haus}\left(R_{1}, R_{2}\right)=O\left(\left\|p_{1}-p_{2}\right\|_{\infty, 2}^{*}\right)$. 
LEMma 13. Let $p_{h}$ be the smoothed density and $R_{h}$ be the associated ridges. Let $\widehat{p}_{h}$ be the KDE based on the observed data $\mathbb{X}_{n}=\left\{X_{1}, \cdots, X_{n}\right\}$ and $\widehat{R}_{h}$ be the estimated ridge. Consider these two conditions:

(T1) (P1-P2) holds for $\widehat{p}_{h}$.

(T2) $\left\|\widehat{p}_{h}-p_{h}\right\|_{\infty, 4}^{*}<s_{0}$ for a small constant $s_{0}$.

Let $\mathcal{X}_{n}=\left\{\mathbb{X}_{n}:(T 1),(T 2)\right.$ holds $\}$. Then, when $n$ is sufficiently large,

$$
\mathbb{P}\left(\mathcal{X}_{n}\right) \geq 1-5 e^{-n h^{d+8} D_{1}},
$$

for some constant $D_{1}$.

Proof For Lemma 13. When $\left\|\widehat{p}_{h}-p_{h}\right\|_{\infty, 4}^{*}$ is sufficiently small (P1-P2) will be satisfied for $\widehat{p}_{h}$. This is because the conditions in (P1-P2) involve the bounds on the eigenvalues of Hessian matrix and the third derivatives around the ridge set.

Let $D_{0}<s_{0}$ be such that

$$
\left\|\widehat{p}_{h}-p_{h}\right\|_{\infty, 4}^{*}<D_{0} \Rightarrow(\mathrm{P} 1-\mathrm{P} 2) \text { valid. }
$$

Then $\mathbb{P}\left(\mathcal{X}_{n}\right) \geq \mathbb{P}\left(\left\|\widehat{p}_{h}-p_{h}\right\|_{\infty, 3}^{*}<D_{0}\right)$. Now

$$
\widehat{p}_{h}(x)-p_{h}(x)=\widehat{p}_{h}(x)-\mathbb{E}\left[\widehat{p}_{h}(x)\right]=\frac{1}{\sqrt{n}} \mathbb{G}_{n}\left(\zeta_{x}\right)
$$

where $\zeta_{x}(y)=\frac{1}{h^{d}} K\left(\frac{x-y}{h}\right)$. Now

$$
\left\|\widehat{p}_{h}-p_{h}\right\|_{\infty, 4}^{*}=\frac{1}{\sqrt{n}} \max \left\{\frac{1}{h^{d+\ell}} \sup _{f \in \mathcal{K}_{\ell}}\left\|\mathbb{G}_{n}(f)\right\|: \ell=0, \cdots, 4\right\},
$$

where $\mathcal{K}_{\ell}$ is the collection of $\ell$-th order partial derivatives for the kernel functions defined in assumption (K2). Recall

$$
\left\|\widehat{p}_{h}-p_{h}\right\|_{\infty}^{(\ell)}=\frac{1}{\sqrt{n} h^{d+\ell}} \sup _{f \in \mathcal{K}_{\ell}}\left\|\mathbb{G}_{n}(f)\right\|
$$

is the supremum norm over $\ell$-th order derivatives for all $x \in \mathbb{R}^{d}$ and

$$
\left\|\widehat{p}_{h}-p_{h}\right\|_{\infty, 4}^{*}=\max \left\{\left\|\widehat{p}_{h}-p_{h}\right\|_{\infty}^{(\ell)}: \ell=0, \cdots, 4\right\} .
$$

We define

$$
P_{\ell}=\mathbb{P}\left(\left\|\widehat{p}_{h}-p_{h}\right\|_{\infty}^{(\ell)}>D_{0}\right)
$$


We recall the Talagrand's inequality (Theorem A.4 in Chernozhukov et al. (2013a); see also Talagrand (1996), Massart (2000) and Gine and Guillou (2002)):

$$
\mathbb{P}\left(\sup _{f \in \mathcal{K}_{\ell}}\left\|\mathbb{G}_{n}(f)\right\|>A h^{d / 2} \sqrt{t}\right) \leq e^{-t}
$$

for some constant $A$. Note that $h^{d / 2}$ comes from $\sigma^{2}=\sup _{f \in \mathcal{K}_{l}} \mathbb{E}\left[f\left(X_{i}\right)^{2}\right] \leq$ $b_{0}^{2} h^{d}$ where $b_{0}$ is the constant envelope by (K2). Note that equation (31) is valid only for a specific interval of $t$ but we will use $t$ in this interval. Hence, combining (29) and (30) and the above yields

$$
\begin{aligned}
P_{\ell} & =\mathbb{P}\left(\left\|\widehat{p}_{h}-p_{h}\right\|_{\infty}^{(\ell)}>D_{0}\right) \\
& =\mathbb{P}\left(\sup _{f \in \mathcal{K}_{\ell}}\left\|\mathbb{G}_{n}(f)\right\|>\sqrt{n h^{2 d+2 \ell}} D_{0}\right) \leq e^{-n h^{d+2 \ell} D_{0} / A}
\end{aligned}
$$

for $\ell=0, \ldots, 4$. We used $t=-n h^{d+2 \ell} D_{0} / A$ in the last inequality.

By definition, $\left\|\widehat{p}_{h}-p_{h}\right\|_{\infty, 3}^{*}>D_{0}$ if and only if one of $\left\|\widehat{p}_{h}-p_{h}\right\|_{\infty}^{(\ell)}>D_{0}$ for $\ell=0, \cdots, 4$. As a result,

$$
\begin{aligned}
\mathbb{P}\left(\mathcal{X}_{n}\right) & \geq \mathbb{P}\left(\left\|\widehat{p}_{h}-p_{h}\right\|_{\infty, 4}^{*}<D_{0}\right) \\
& \geq 1-P_{0}-P_{1}-P_{2}-P_{3}-P_{4} \\
& \geq 1-5 e^{-n h^{d+8} D_{1}}
\end{aligned}
$$

for some constant $D_{1}$. We used $\ell=4$ in the last inequality to bound each $\ell$. This completes the proof.

Lemma 14. Let $R_{1}, R_{2}$ be two closed, non self-intersecting curves with positive reach. If

$$
\operatorname{Haus}\left(R_{1}, R_{2}\right)<(2-\sqrt{2}) \min \left\{\operatorname{reach}\left(R_{1}\right), \operatorname{reach}\left(R_{2}\right)\right\},
$$

then

$$
\operatorname{dist}_{\Pi}\left(R_{2}, R_{1}\right)=\operatorname{dist}_{\Pi}\left(R_{1}, R_{2}\right)=\operatorname{Haus}\left(R_{1}, R_{2}\right) .
$$

ProOF FOR LEMMA 14. Observe the following fact:

(F3) $\operatorname{dist}_{\Pi}\left(R_{1}, R_{2}\right)=\operatorname{Haus}\left(R_{1}, R_{2}\right)$ if the projection $\pi_{R_{1}}$ defined on $R_{2}$ is onto. i.e.

$$
\pi_{R_{1}}\left(R_{2}\right)=\left\{\pi_{R_{1}}(x): x \in R_{2}\right\}=R_{1}
$$

imsart-aos ver. 2013/03/06 file: ridgeAOSsupp.tex date: March 10, 2015 
To see (F3), recall the definition,

$$
\operatorname{dist}_{\Pi}\left(R_{1}, R_{2}\right)=\sup _{x \in R_{2}} d\left(x, R_{1}\right)
$$

and hence,

$$
R_{2} \subset R_{1} \oplus \operatorname{dist}_{\Pi}\left(R_{1}, R_{2}\right) .
$$

If $\pi_{R_{1}}\left(R_{2}\right)=R_{1}$, we have

$$
R_{1}=\pi_{R_{1}}\left(R_{2}\right) \subset R_{2} \oplus \operatorname{dist}_{\Pi}\left(R_{1}, R_{2}\right) .
$$

Thus, $\operatorname{dist}_{\Pi}\left(R_{1}, R_{2}\right)$ satisfies the condition for $\operatorname{Haus}\left(R_{1}, R_{2}\right)$ which proves (F3).

Now we prove that the condition

$$
\operatorname{Haus}\left(R_{1}, R_{2}\right)<(2-\sqrt{2}) \min \left\{\operatorname{reach}\left(R_{1}\right), \operatorname{reach}\left(R_{2}\right)\right\}
$$

implies the projection $\pi_{R_{1}}$ defined on $R_{2}$ is onto. This can be proved by a similar argument as in Theorem 1 in Chazal et al. (2007). Although Chazal et al. (2007) proved the case for $d-1$ manifold in $\mathbb{R}^{d}$, the same idea can be generalized to ridges. Note that we can exchange $R_{1}, R_{2}$ in (F3). Thus, we have proved the assertion.

Consider two densities $p_{1}, p_{2}$ satisfying conditions (A1), (P1-P2). Let $R_{1}, R_{2}$ be the density ridges for $p_{1}, p_{2}$ respectively. We assume conditions (K1-K2). Define

$$
\mathcal{F}_{k}=\left\{\mathbf{w}^{T} f_{x, k}: \mathbf{w} \in \mathbb{R}^{d},\|\mathbf{w}\|=1, x \in R_{k}\right\}, k=1,2
$$

where

$$
f_{x, k}=\frac{1}{\sqrt{h^{d+2}}} N_{k}(x) H_{N, k}(x)^{-1} N_{k}(x)^{T}(\nabla K)\left(\frac{x-y}{h}\right) \in \mathbb{R}^{d} .
$$

Note that we have two indices $x, \mathrm{w}$ for each element in $\mathcal{F}_{1}$ and $\mathcal{F}_{2}$. The first index $x$ is the location and the second index $\mathbf{w}$ is the direction. $N_{k}(x)$ is the normal matrix (as $x \in R_{k}$, its column space is the normal space) defined by Lemma 2 at $x$ and $H_{N, k}(x)$ is the subspace Hessian in the columns space of $N_{k}(x)$.

For any function $f \in \mathcal{F}_{k}$, we define the operator $\Xi_{k}$ that maps $f$ to its location' $x$. That is, for $f=\mathbf{w}^{T} f_{x, k} \in \mathcal{F}_{k}$ with $f_{x, k}$ defined in (36), $\Xi_{k}(f)=x$. Similarly, we define the operator $\Upsilon_{k}$ that maps $f$ to the direction the associated 'direction' $\mathbf{w}$. i.e. for $f=\mathbf{w}^{T} f_{x, k} \in \mathcal{F}_{k}$ with $f_{x, k}$ defined in $(36), \Upsilon_{k}(f)=\mathbf{w}$. 
Lemma 17 (Gaussian comparison on two ridges). When $\left\|p_{1}-p_{2}\right\|_{\infty, 3}^{*}$ is sufficiently small, we have

$\sup _{t}\left|\mathbb{P}\left(\|\mathbb{B}\|_{\mathcal{F}_{1}}<t\right)-\mathbb{P}\left(\|\mathbb{B}\|_{\mathcal{F}_{2}}<t\right)\right|=O\left(\left(\left\|p_{1}-p_{2}\right\|_{\infty, 3}^{*}+\operatorname{Haus}\left(R_{1}, R_{2}\right) / h\right)^{1 / 3}\right)$.

Proof for Lemma 17. We first recall a Gaussian comparison theorem.

Theorem 18 (Theorem 2 in Chernozhukov et al. (2013b)). Let $V=$ $\left(V_{1}, \cdots, V_{N}\right) \stackrel{d}{=} N\left(0, \Sigma^{V}\right)$ and $W=\left(W_{1}, \cdots, W_{N}\right) \stackrel{d}{=} N\left(0, \Sigma^{W}\right)$. Define

$$
\Delta=\max _{1 \leq j, k \leq N}\left|\Sigma_{j, k}^{V}-\Sigma_{j, k}^{W}\right| .
$$

Suppose that $\min _{j} \Sigma_{j j}^{W}>0$. Then

$$
\sup _{t}\left|\mathbb{P}\left(\max _{1 \leq i \leq N} V_{i}<t\right)-\mathbb{P}\left(\max _{1 \leq i \leq N} W_{i}<t\right)\right| \leq C \Delta^{1 / 3}(1 \vee \log (N / \Delta))^{2 / 3},
$$

where $C$ depends only $\min _{j} \Sigma_{j j}^{W}$, and $\max _{j} \Sigma_{j j}^{W}$.

Our strategy is to show that for any net for both classes, we can find two finite sub-classes $\breve{\mathcal{F}}_{1} \subset \mathcal{F}_{1}, \breve{\mathcal{F}}_{2} \subset \mathcal{F}_{2}$ such that

1. $\breve{\mathcal{F}}_{1}, \breve{\mathcal{F}}_{2}$ contain the nets for $\mathcal{F}_{1}, \mathcal{F}_{2}$ respectively.

2. We can find a one-to-one correspondence between elements in $\breve{\mathcal{F}}_{1}, \breve{\mathcal{F}}_{2}$ such that each element in the second space assigned to an element in the first space is close.

This is accomplished by Lemma 19. Then we use Theorem 18 for the pair of elements and bound the difference in the covariance matrices (Lemma 20) to obtain the desired bound. More explicitly, we want to find $\breve{\mathcal{F}}_{1}=\left\{f_{1}, \cdots, f_{N}\right\} \subset$ $\mathcal{F}_{1}$ and $\breve{\mathcal{F}}_{2}=\left\{g_{1}, \cdots, g_{N}\right\} \subset \mathcal{F}_{2}$ such that

$$
\begin{aligned}
\sup _{t}\left|\mathbb{P}\left(\| \mathbb{B}||_{\mathcal{F}_{1}}<t\right)-\mathbb{P}\left(\|\mathbb{B}\|_{\mathcal{F}_{2}}<t\right)\right| & \leq \underbrace{\sup _{t}\left|\mathbb{P}\left(\|\mathbb{B}\|_{\mathcal{F}_{1}}<t\right)-\mathbb{P}\left(\|\mathbb{B}\|_{\breve{\mathcal{F}}_{1}}<t\right)\right|}_{I V} \\
& +\underbrace{\sup _{t}\left|\mathbb{P}\left(\|\mathbb{B}\|_{\breve{\mathcal{F}}_{1}}<t\right)-\mathbb{P}\left(\|\mathbb{B}\|_{\breve{\mathcal{F}}_{2}}<t\right)\right|}_{V} \\
& +\underbrace{\sup _{t}\left|\mathbb{P}\left(\|\mathbb{B} \mid\|_{\mathcal{F}_{2}}<t\right)-\mathbb{P}\left(\|\mathbb{B}\|_{\breve{\mathcal{F}}_{2}}<t\right)\right|}_{V I}
\end{aligned}
$$


with $I V, V, V I$ are bounded. We can bound $I V$ and $V I$ if we pick $\breve{\mathcal{F}}_{1}, \breve{\mathcal{F}}_{2}$ contains the covering sets. So in what follows we will bound $V$ from two covering sets.

By condition (K2), the partial derivative of kernel functions have a constant envelope $b_{0}$ and since (A1) holds for both $p_{1}, p_{2}$, the matrix $N_{k}(x)$ is uniformly bounded for $x \in R_{k}$. This also implies $H_{N, k}^{-1}(x)$ is uniformly bounded. Hence, there exists a constant envelope $b_{2}<\infty$ for both $\mathcal{F}_{1}$ and $\mathcal{F}_{2}$.

We define the uniform covering numbers for both functional spaces by

$$
\begin{aligned}
& N_{1}(\epsilon)=\sup _{Q} N\left(\mathcal{F}_{1}, \mathcal{L}^{2}(Q), b_{2} \epsilon\right) \\
& N_{2}(\epsilon)=\sup _{Q} N\left(\mathcal{F}_{2}, \mathcal{L}^{2}(Q), b_{2} \epsilon\right) .
\end{aligned}
$$

These can differ. Note that the $\mathcal{L}^{2}(Q)$ norm of $f$ is $\int|f(x)|^{2} d Q(x)$.

For each $\epsilon>0$, let $N=N_{1}(\epsilon)+N_{2}(\epsilon)$. By Lemma 19, we can find $\breve{\mathcal{F}}_{1}=$ $\left\{f_{1}, \cdots, f_{N}\right\} \subset \mathcal{F}_{1}$ and $\breve{\mathcal{F}}_{2}=\left\{g_{1}, \cdots, g_{N}\right\} \subset \mathcal{F}_{2}$ such that $\breve{\mathcal{F}}_{1}$ is an $\epsilon$-covering set for $\mathcal{F}_{1}$ and $\breve{\mathcal{F}}_{2}$ is also an $\epsilon$-covering set for $\mathcal{F}_{2}$. Furthermore, for each pair $f_{j}, g_{j}$ constructed from above, the corresponding location $\Xi_{1}\left(f_{j}\right), \Xi_{2}\left(g_{j}\right)$ satisfies $\left\|\Xi_{1}\left(f_{j}\right)-\Xi_{2}\left(g_{j}\right)\right\| \leq \operatorname{Haus}\left(R_{1}, R_{2}\right)$.

Define the following two random vectors:

$$
\begin{gathered}
V=\left(V_{1}, \cdots, V_{N}\right)=\left(\mathbb{B}\left(f_{1}\right), \cdots, \mathbb{B}\left(f_{N}\right)\right) \stackrel{d}{=} N\left(0, \Sigma_{1}\right) \\
W=\left(W_{1}, \cdots, W_{N}\right)=\left(\mathbb{B}\left(g_{1}\right), \cdots, \mathbb{B}\left(g_{N}\right)\right) \stackrel{d}{=} N\left(0, \Sigma_{2}\right) .
\end{gathered}
$$

Let $\Delta_{12}=\left\|\Sigma_{1}-\Sigma_{2}\right\|_{\infty}$. Then Theorem 18 implies

$$
\begin{aligned}
\sup _{t} \mid \mathbb{P}\left(\|\mathbb{B}\|_{\breve{\mathcal{F}}_{1}}<t\right) & -\mathbb{P}\left(\| \mathbb{B}||_{\breve{\mathcal{F}}_{2}}<t\right) \mid \\
& =\sup _{t}\left|\mathbb{P}\left(\max _{1 \leq i \leq N} V_{i}<t\right)-\mathbb{P}\left(\max _{1 \leq i \leq N} W_{i}<t\right)\right| \\
& =O\left(\Delta_{12}^{1 / 3}\right)
\end{aligned}
$$

To bound $\Delta_{12}^{1 / 3}$, we consider the pair of function $f_{j}, g_{j}$. By Lemma 20,

$$
\left\|\mathbb{B}\left(f_{j}\right)-\mathbb{B}\left(g_{j}\right)\right\| \leq C_{1}\left(\left\|p_{1}-p_{2}\right\|_{\infty, 3}^{*}+\operatorname{Haus}\left(R_{1}, R_{2}\right) / h\right)
$$

for all $j=1, \cdots, N$ and some constant $C_{1}$.

Hence, for any $i, j$ we have

$$
\begin{aligned}
& \left|\operatorname{Cov}\left(V_{i}, V_{j}\right)-\operatorname{Cov}\left(W_{i}, W_{j}\right)\right| \leq C_{1}\left(\left\|p_{1}-p_{2}\right\|_{\infty, 3}^{*}+\operatorname{Haus}\left(R_{1}, R_{2}\right) / h\right) \\
& \text { imsart-aos ver. 2013/03/06 file: ridgeAOSsupp.tex date: March 10, } 2015
\end{aligned}
$$




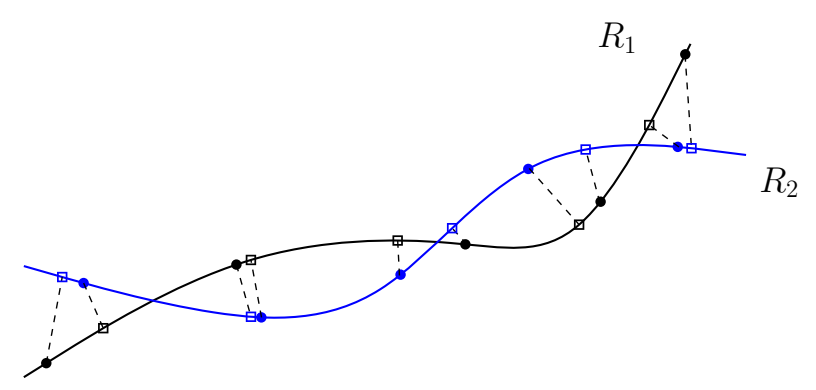

FIG 1. A plot for constructing $\breve{\mathcal{F}}_{1}, \breve{\mathcal{F}}_{2}$. Black dots are the corresponding points of covering set for $\mathcal{F}_{1}$ and blue dots are that for $\mathcal{F}_{2}$. Black boxes are projections from blue dots onto $R_{1}$ while blue boxes are projections from black dots onto $R_{2}$. Note that since the projections must have distance less than the Hausdorff distance between two sets; each pair of box-dot has distance less than $\operatorname{Haus}\left(R_{1}, R_{2}\right)$ and the dots contain all covering sets. The collection of all black dots and boxes corresponds to $\breve{\mathcal{F}}_{1}$, while all blue dots and boxes consist of $\breve{\mathcal{F}}_{2}$,.

This leads to the uniform bound for all elements in the covariance matrix. As a result, we have

$$
\Delta_{12} \leq C_{1}\left(\left\|p_{1}-p_{2}\right\|_{\infty, 3}^{*}+\operatorname{Haus}\left(R_{1}, R_{2}\right) / h\right) .
$$

Recalling (40) and using the above result,

$$
\begin{aligned}
\sup _{t} \mid \mathbb{P}\left(\|\mathbb{B}\|_{\breve{\mathcal{F}}_{1}}<t\right) & -\mathbb{P}\left(\|\mathbb{B}\|_{\breve{\mathcal{F}}_{2}}<t\right) \mid \\
& =O\left(\left(\left\|p_{1}-p_{2}\right\|_{\infty, 3}^{*}+\operatorname{Haus}\left(R_{1}, R_{2}\right) / h\right)^{1 / 3}\right) .
\end{aligned}
$$

This bounds $V$, and hence we obtain our result.

Lemma 19. Assume the same notation and assumptions as Lemma 17. Let $N_{1}=N_{1}(\epsilon), N_{2}=N_{2}(\epsilon)$ be the uniform $\epsilon$-covering number of $\mathcal{F}_{1}, \mathcal{F}_{2}$ respectively and $N=N(\epsilon)=N_{1}(\epsilon)+N_{2}(\epsilon)$. Then for any distribution $Q$ and $\epsilon$-covering sets for $\mathcal{F}_{1}, \mathcal{F}_{2}$, we can find $\breve{\mathcal{F}}_{1}=\left\{f_{1}, \cdots, f_{N}\right\} \subset \mathcal{F}_{1}$ and $\breve{\mathcal{F}}_{2}=\left\{g_{1}, \cdots, g_{N}\right\} \subset \mathcal{F}_{2}$ such that

1. $\breve{\mathcal{F}}_{1}, \breve{\mathcal{F}}_{2}$ contain the $\epsilon$-covering sets of $\mathcal{F}_{1}$ and $\mathcal{F}_{2}$ respectively.

2. For each $i,\left\|\Xi_{1}\left(f_{i}\right)-\Xi_{2}\left(g_{i}\right)\right\| \leq \operatorname{Haus}\left(R_{1}, R_{2}\right)$.

3. For each $i, \Upsilon_{1}\left(f_{i}\right)=\Upsilon_{2}\left(g_{i}\right)$.

Proof. For any given $\epsilon$ and a given $Q$, we can find a covering set $D_{1}$ for $\mathcal{F}_{1}$ such that $\# D_{1}=N_{1}$; let the elements of $D_{1}$ be $f_{1}^{0}, \cdots, f_{N_{1}}^{0}$. Similarly, we can find $D_{2}$ for $\mathcal{F}_{2}$ such that $D_{2}=\left\{g_{1}^{0}, \cdots, g_{N_{2}}^{0}\right\}$. For each $f_{i}^{0} \in D_{1}$, it has 
two indexes: location $\Xi_{1}\left(f_{i}^{0}\right) \in R_{1}$ and the direction $\Upsilon_{1}\left(f_{i}\right)$. Similarly, each $g_{i}^{0}$ corresponds to a location $\Xi_{2}\left(g_{i}^{0}\right) \in R_{2}$ and a direction $\Upsilon_{2}\left(g_{i}\right)$. Figure 1 shows an example for this proof. The black dots are $\Xi_{1}\left(f_{1}^{0}\right), \cdots, \Xi_{1}\left(f_{N_{1}}^{0}\right)$ and blue dots are $\Xi_{2}\left(g_{1}^{0}\right), \cdots, \Xi_{2}\left(g_{N_{2}}^{0}\right)$.

Recall that $\pi_{A}(x)$ is the projection for $x$ onto a set $A$. For each $i, \pi_{R_{2}}\left(\Xi_{1}\left(f_{i}^{0}\right)\right)$ is one point on $R_{2}$. Notice that this location and the direction vector $\Upsilon_{1}\left(f_{i}^{0}\right)$ corresponds to one element in $\mathcal{F}_{2}$. That is, there exists an element $g_{i}^{\prime} \in \mathcal{F}_{2}$ such that

$$
\begin{aligned}
\Xi_{2}\left(g_{i}^{\prime}\right) & =\pi_{R_{2}}\left(\Xi_{1}\left(f_{i}^{0}\right)\right) \\
\Upsilon\left(g_{i}^{\prime}\right) & =\Upsilon_{1}\left(f_{i}^{0}\right) .
\end{aligned}
$$

We define $\Pi_{\mathcal{F}_{2}}\left(f_{i}^{0}\right)=g_{i}^{\prime}$; that is, for any $f_{i}^{0}$, we pick the projected point from the location of $f_{i}^{0}$ onto $R_{2}$ and the same direction vector as two indexes to select one element $\Pi_{\mathcal{F}_{2}}\left(f_{i}^{0}\right)$ in $\mathcal{F}_{2}$. Similarly, we can define $\Pi_{\mathcal{F}_{1}}\left(g_{i}^{0}\right) \in \mathcal{F}_{1}$. In Figure 1, each black box is one $\pi_{R_{1}}\left(\Xi_{2}\left(g_{i}^{0}\right)\right)$ which is an projection from a blue dots while each blue box is a projection from a black dot.

Now we define

$$
f_{i}= \begin{cases}f_{i}^{0} & 1 \leq i \leq N_{1} \\ \Pi_{\mathcal{F}_{1}}\left(g_{i-N_{1}}^{0}\right) & N_{1}+1 \leq i \leq N_{1}+N_{2}\end{cases}
$$

and

$$
g_{i}= \begin{cases}\Pi_{\mathcal{F}_{2}}\left(f_{i}^{0}\right) & 1 \leq i \leq N_{1} \\ g_{i-N_{1}}^{0} & N_{1}+1 \leq i \leq N_{1}+N_{2}\end{cases}
$$

Each pair $\left(f_{i}, g_{i}\right)$ is a projection between $R_{1}, R_{2}$ so that the distance between the locations of them must be less than $\operatorname{Haus}\left(R_{1}, R_{2}\right)$. The collection of $f_{i}$ contains all $f_{i}^{0}$ which contains the covering set $D_{1}$ and similarly, the collection of $g_{i}$ contains $D_{2}$. So the collection $\breve{\mathcal{F}}_{1}=\left\{f_{1}, \cdots, f_{N}\right\} \subset \mathcal{F}_{1}$ and $\breve{\mathcal{F}}_{2}=\left\{g_{1}, \cdots, g_{N}\right\} \subset \mathcal{F}_{2}$ have the desire properties.

We say that a Gaussian process $\mathbb{B}_{p}$ is generated by a density $p$ if for any two elements $f_{1}, f_{2}$ in a collection of function $\mathcal{F}$ that is pre-Gaussian, we have

$$
\operatorname{Cov}\left(\mathbb{B}_{p}\left(f_{1}\right), \mathbb{B}_{p}\left(f_{2}\right)\right)=\mathbb{E}\left[f_{1}(Z) f_{2}(Z)\right]-\mathbb{E}\left[f_{1}(Z)\right] \mathbb{E}\left[f_{2}(Z)\right]
$$

with $Z$ being sampled from the density $p$.

Lemma 20. Assume the same assumptions as Lemma 17. Let $\mathbb{B}_{p}$ be the Gaussian process generated by a density $p \in \mathbf{C}^{4}$. Let $\mathcal{I}_{12}$ be the set of pairs of functions satisfying:

1. $\left\|\Xi_{2}\left(f_{1}\right)-\Xi_{1}\left(f_{2}\right)\right\| \leq \operatorname{Haus}\left(R_{1}, R_{2}\right)$ 
2. $\Upsilon_{1}\left(f_{1}\right)=\Upsilon_{2}\left(f_{2}\right)$.

When $\left\|p_{1}-p_{2}\right\|_{3, \infty}^{*}$ is sufficiently small, we have

$$
\sup _{f_{i} \in \mathcal{F}_{i}, \mathcal{I}_{12}}\left|\mathbb{B}_{p}\left(f_{1}\right)-\mathbb{B}_{p}\left(f_{2}\right)\right| \leq C_{1}\left(|| p_{1}-p_{2} \|_{3, \infty}^{*}+\operatorname{Haus}\left(R_{1}, R_{2}\right) / h\right),
$$

where $C_{1}$ is a constant.

Proof. We begin the proof by recalling the definition of $\mathcal{F}_{k}$ from equation $(35)$ and (36):

$$
\mathcal{F}_{k}=\left\{\mathbf{w}^{T} f_{x, k}: \mathbf{w} \in \mathbb{R}^{d},\|\mathbf{w}\|=1, x \in R_{k}\right\}, k=1,2
$$

where each $f_{x, k}$ is defined as

$$
f_{x, k}(y)=\frac{1}{\sqrt{h^{d+2}}} N_{k}(x) H_{N, k}(x)^{-1} N_{k}(x)^{T}(\nabla K)\left(\frac{x-y}{h}\right) \in \mathbb{R}^{d}
$$

and $N_{k}(x)$ is the matrix whose columns are orthonormal basis for the normal space of $R_{k}$ at $x$ and $H_{N, k}(x)$ is the subspace Hessian in the columns space of $N_{k}(x)$. Hence, we see that each element of $\mathcal{F}_{k}$ is the multiplication of $\mathbf{w}, N_{k}(x), H_{N, k}(x)^{-1}$ and $(\nabla K)\left(\frac{x-y}{h}\right)$.

Consider a pair of functions $f_{1} \in \mathcal{F}_{1}, f_{2} \in \mathcal{F}_{2}$ satisfying condition $\mathcal{I}_{12}$. i.e. $\left|\Xi_{1}\left(f_{1}\right)-\Xi_{2}\left(f_{2}\right)\right| \leq \operatorname{Haus}\left(R_{1}, R_{2}\right)$ and $\mathbf{w}_{1}=\mathbf{w}_{2}$. Recalling from Lemma 4 , we denote

$$
W_{p_{k}}(x)=N_{k}(z) H_{N, k}^{-1}(z) N_{k}(z)^{T} .
$$

The function $f_{k}(y)$ can be written as

$$
f_{k}(y)=\mathbf{w}_{k}^{T} W_{p_{k}}\left(\Xi_{k}\left(f_{k}\right)\right)(\nabla K)\left(\frac{\Xi_{k}\left(f_{k}\right)-y}{h}\right) .
$$

Our goal is to bound $\left|f_{1}(y)-f_{2}(y)\right|$. Because $\mathbf{w}_{1}=\mathbf{w}_{2}$, the difference in fact contains only two parts: $W$ and $(\nabla K)$. i.e.

(A) $\left\|W_{p_{1}}\left(\Xi_{1}\left(f_{1}\right)\right)-W_{p_{2}}\left(\Xi_{2}\left(f_{2}\right)\right)\right\|_{\max }$

(B) $\left\|(\nabla K)\left(\frac{\Xi_{1}\left(f_{1}\right)-y}{h}\right)-(\nabla K)\left(\frac{\Xi_{2}\left(f_{2}\right)-y}{h}\right)\right\|_{\max }$.

To bound (A), recall that from Lemma 4 and Lemma 2, $W(p)$ is uniquely defined by $p$ and is Lipschitz in the following sense

$$
\begin{aligned}
\left\|W_{p_{1}}(x)-W_{p_{2}}(x)\right\|_{\max } & \leq A_{3}\left\|p_{1}-p_{2}\right\|_{\infty, 3}^{*} \\
\left\|W_{p_{1}}(x)-W_{p_{1}}(y)\right\|_{\max } & \leq A_{4}\left(\left\|p_{1}^{(3)}\right\|_{\infty}+\left\|p_{1}^{(4)}\right\|_{\infty}\right)^{2}\|x-y\| \\
& \leq A_{5}\|x-y\|
\end{aligned}
$$

imsart-aos ver. 2013/03/06 file: ridgeAOSsupp.tex date: March 10, 2015 
for some constants $A_{3}, A_{4}, A_{5}$. Note that we absorb $\left(\left\|p_{1}^{(3)}\right\|_{\infty}+\left\|p_{1}^{(4)}\right\|_{\infty}\right)^{2}$ into the constant $A_{5}$ by assumption (A1). Now by the fact that $\mid \Xi_{1}\left(f_{1}\right)-$ $\Xi_{2}\left(f_{2}\right) \mid \leq \operatorname{Haus}\left(R_{1}, R_{2}\right) \leq\left\|p_{1}-p_{2}\right\|_{\infty, 2}^{*}$ from Theorem 12, we conclude that

$$
\left\|W_{p_{1}}\left(\Xi_{1}\left(f_{1}\right)\right)-W_{p_{2}}\left(\Xi_{2}\left(f_{2}\right)\right)\right\|_{\max } \leq A_{6}\left(\left\|p_{1}-p_{2}\right\|_{\infty, 3}^{*}\right)
$$

for some constant $A_{6}$.

To bound (B), we use Taylor's theorem:

$$
\nabla K\left(\frac{\Xi_{1}\left(f_{1}\right)-y}{h}\right)-\nabla K\left(\frac{\Xi_{2}\left(f_{2}\right)-y}{h}\right)=B_{0} \times\left(\frac{\Xi_{1}\left(f_{1}\right)-\Xi_{2}\left(f_{2}\right)}{h}\right),
$$

where

$$
B_{0}=\int_{0}^{1}(\nabla \nabla K)\left(\frac{\Xi_{2}\left(f_{2}\right)+\left(\Xi_{1}\left(f_{1}\right)-\Xi_{2}\left(f_{2}\right)\right) t-y}{h}\right) d t
$$

By assumption (K2), $\left\|\nabla \nabla K\left(\frac{x-y}{h}\right)\right\|_{\max } \leq b_{0}$ for all $x, y$ so that $\left\|B_{0}\right\|_{\max } \leq$ $b_{0}$ which implies that

$$
\begin{aligned}
\left\|\nabla K\left(\frac{\Xi_{1}\left(f_{1}\right)-y}{h}\right)-\nabla K\left(\frac{\Xi_{2}\left(f_{2}\right)-y}{h}\right)\right\|_{\max } & \leq b_{0}\left\|\frac{\Xi_{1}\left(f_{1}\right)-\Xi_{2}\left(f_{2}\right)}{h}\right\| \\
& \leq \frac{b_{0}}{h} \operatorname{Haus}\left(R_{1}, R_{2}\right) .
\end{aligned}
$$

The result follows from (45) and (46).

\section{References.}

R. Bhatia. Matrix Analysis. Springer, 1997.

F. Chazal, A. Lieutier, and J. Rossignac. Normal-map between normal-compatible manifolds. Int. J. Comput. Geometry Appl., 17(5):403-421, 2007. URL http://dblp. uni-trier.de/db/journals/ijcga/ijcga17.html\#ChazalLR07.

$\mathrm{X}$. Chen, H. Qi, and P. Tseng. Analysis of nonsmooth symmetric-matrix-valued functions with applications to semidefinite complementarity problems. SIAM J. OPTIM., 2003.

V. Chernozhukov, D. Chetverikov, and K. Kato. Anti-concentration and honest adaptive confidence bands. arXiv:1303.7152, 2013a.

V. Chernozhukov, D. Chetverikov, and K. Kato. Comparison and anti-concentration bounds for maxima of gaussian random vectors. arXiv:1301.4807, 2013b.

H. Federer. Curvature measures. Trans. Am. Math. Soc, 93, 1959.

C. R. Genovese, M. Perone-Pacifico, I. Verdinelli, and L. Wasserman. Nonparametric ridge estimation. arXiv:1212.5156v1, 2012.

E. Gine and A. Guillou. Rates of strong uniform consistency for multivariate kernel density estimators. In Annales de l'Institut Henri Poincare (B) Probability and Statistics, 2002.

P. Massart. About the constants in talagrand's concentration inequalities for empirical processes. The Annals of Probability, 2000. 
G. W. Stewart. On the perturbation of pseudo-inverses, projections and linear least squares problems. SIAM Review, 1977.

M. Talagrand. Newconcentration inequalities in product spaces. Invent. Math, 1996.

Department of Statistics

Carnegie Mellon University

5000 Forbes Ave.

Pittsburgh, PA 15213

E-MAIL: yenchic@andrew.cmu.edu 